\title{
Study on the Influence of Sand Core Compactness on Surface Entropy considering Engineering Disturbance
}

\author{
Ding-li Su, ${ }^{1}$ Hang Chen, ${ }^{2}$ Xuemiao Xu, ${ }^{3}$ Mengxiong Tang, ${ }^{4}$ and He-song $\mathrm{Hu}{ }^{1}{ }^{1}$ \\ ${ }^{1}$ Guangzhou Institute of Building Science Group Co., Ltd., Guangzhou 510440, China \\ ${ }^{2}$ Guangzhou Testing Centre of Construction Quality and Safety Co., Ltd., Guangzhou 510440, China \\ ${ }^{3}$ South China University of Technology, Guangzhou 511442, China \\ ${ }^{4}$ Guangzhou Municipal Construction Group Co., Ltd., Guangzhou 510030, China \\ Correspondence should be addressed to He-song Hu; hesonghu79@126.com
}

Received 4 April 2021; Revised 7 July 2021; Accepted 13 July 2021; Published 27 July 2021

Academic Editor: Tianshou Ma

Copyright (C) 2021 Ding-li Su et al. This is an open access article distributed under the Creative Commons Attribution License, which permits unrestricted use, distribution, and reproduction in any medium, provided the original work is properly cited.

\begin{abstract}
In order to explore the correlation between the compactness of sand core samples and its surface image features and to provide the basis for rapid identification and recognition of core samples in engineering investigation, a typical image data set of sand core samples disturbed by drilling construction in practical engineering has been established, using Python language to compile algorithm to calculate one-dimensional entropy and two-dimensional entropy of 60 groups of sand core samples with different densities. The influence of different sand core compactness on surface entropy characteristics was discussed, and the following conclusions were obtained: (1) Affected by drilling construction and disturbance, the looser the sand core surface particles are, the worse the sorting is and the more irregular the shape characteristics are. There is a close relationship between grain texture and compactness. (2) The calculation results of sand image entropy of one-dimensional entropy and two-dimensional entropy showed that the entropy value of loose, slightly dense, and medium dense sand images is positively correlated with the compactness of sand. (3) The maximum variance of two-dimensional entropy of loose, slightly dense, and medium dense sand image in the same borehole is less than 0.09 , and the data variance amplification effect of two-dimensional entropy of image is mainly between different boreholes. (4) The dense feature of core sample structure forms an ordered structure with a gray change boundary, which increases the roughness of the image and leads to the increase of entropy. The two-dimensional entropy reveals the internal correlation mechanism of the influence of the engineering state on the surface structure of sand more clearly than the onedimensional entropy and more effectively characterizes the dense degree of sand particles. (5) Using two-dimensional entropy to judge the compactness of sand image in the same borehole, the data fluctuation is small, and the algorithm is stable and reliable. The research results have reference values for the detection and analysis of sand sample density in geotechnical engineering investigation.
\end{abstract}

\section{Introduction}

Core samples of geotechnical engineering investigation are obtained by drilling. Core identification is the core step of engineering investigation. The accuracy and efficiency of identification directly affect the progress of the project. At present, the core sample identification adopts engineers with rich geological survey experience to conduct artificial discrimination on its surface characteristics, such as low intelligence and strong subjective dependence, and it is not in line with the development requirements of national network power strategy for transformation and upgrading of the survey industry [1-8]. It is urgent to use computer technology to speed up the identification of core samples.

Visual recognition technology is developed with the development of image recognition technology in recent years. Visual object representation based on local features has the characteristics of strong representation ability and robustness to image occlusion and background confusion, which has attracted great attention in recent years. According to the statistical modeling and discriminant learning of local features, the change law of local features can 
be measured and visual objects can be more accurately represented $[9,10]$. At home and abroad, there are many previous research achievements in geotechnical identification, mainly focusing on color features and texture features. Because the color feature of rock and soil is the most obvious, the early image recognition starts from the color feature recognition. Niekum et al. [11] studied the visual recognition segmentation, detection, and classification of desert rocks based on color. In recent years, with the improvement of computer computing power and the rapid development of color recognition technology combined with artificial neural network, many computing models have been generated; for example, Wang and Wang [12] studied neural network algorithm and TensorFlow and the convolution neural network model of rock slope image set analysis is established. Depending on the color features, different types of rock and soil with obvious color differences can be quickly distinguished. However, the colors of rock and soil with different compactness or density are similar, and it is difficult to further subdivide, which is necessary to combine with the surface texture features. Texture features mainly include the spatial distribution of the geometric lines and shape features of the surface. The research on the recognition of texture features is restricted by the complexity of the features. The accuracy of recognition has been rapidly improved after the improvement of the computational power of the computer neural network. Researchers mainly analyzed the texture features through neural network, fractal theory, optimization calculation function, and other methods. Hong et al. [13] put forward a method to quantitatively determine the geological strength index based on image processing, fractal theory, and artificial neural network using the image of rock joint surface. Singh et al. [14] constructed neural network to process and analyze basalt mineral images and realized effective recognition of basalt mineral texture. These works belong to supervised deep network learning, which needs a lot of data. There is a positive correlation between the calculation accuracy and the characteristics of training samples. However, texture features usually have the characteristics of diversity and small sample nature. Although researchers improve the learning sample size through data expansion and data enhancement, the improvement of accuracy is limited. Therefore, unsupervised feature learning methods such as evolutionary algorithm and clustering algorithm also attract researchers' attention. Shu et al. [15] proposed an unsupervised feature learning method to learn the representation of rock image. Zhang et al. [16] proposed rock CT based on clustering analysis algorithm, image segmentation, and quantization method. Researchers found that the accuracy of unsupervised learning method is greatly affected by the representation of the texture features of the collected samples. Ke et al. [17] used variogram as texture calculation function to extract image texture, combined with original spectral data to classify lithology. In the above research, the standard rock and soil sample slice database is generally used, and the database is established by using the disturbed rock and soil core image of engineering investigation site for texture feature analysis, which is rarely reported.
Compactness is the most important analysis parameter of sand core in engineering investigation. Accurate compactness analysis needs a precise instrument test. Engineering researchers usually use standard penetration test blow count to determine sand compactness according to code for geotechnical engineering investigation (GB 500212001). Many scholars have studied the relationship among standard penetration, particle size, density, and compactness of sand through experiments. For example, Meng et al. [18] have studied the influence of relative compactness on dynamic shear modulus and damping ratio of clay sand. Misko and Kenji [19] attempted to correlate the penetration resistance and relative density by accounting for the grain size properties of soils. In addition to geotechnical tests, researchers have applied digital image recognition technology to the analysis of sand structure characteristics. In 1996, Wightman et al. [20] pointed out that image analysis is used to determine the mean, mode, standard deviation, variance, and skewness of local composition for a large number of locations in the mixtures, providing an effective method for performing a detailed quantitative characterization of the mixture structure. In recent years, with the rapid development of computer image recognition technology, it has been applied to smart cities, smart buildings, smart construction sites, and other fields. Zou et al. [21] used eight neighborhood search and clustering methods to realize the connected domain search and sand particle statistics for the digital image of target sand, the same group of dyed particles. Combined with the geotechnical test results, the change relationship between the particle statistics and the corresponding sand compactness was established. However, the size and composition of particles were counted in the test method, which did not characterize the spatial distribution of particles. The test samples are self-made indoor samples, and the in situ coring samples for engineering investigation are not used. Zhou et al. [22] studied the relationship between sand particle morphology and packing density. Combined with digital image analysis technology, eight particle shape feature parameters are extracted, and a theoretical model of compressible compactness correction is proposed. It shows that the particle shape feature extraction from image can be well correlated with compactness; however, the theoretical model needs more parameters to be extracted and has high computational complexity.

In the process of geotechnical engineering investigation and construction, the surface shape and texture characteristics of sand are greatly affected by the compactness. Based on the analysis of the above research progress, compactness, as an implicit attribute affected by both color and texture, is difficult to obtain high-precision judgment results directly through single color feature recognition and texture feature recognition. Therefore, it is necessary to seek a comprehensive characterization variable for classification and identification of sand core compactness. Entropy is a measure of the disorder degree of information system, and it is also a comprehensive expression of image color (gray) and texture (spatial distribution) features. Many researchers use entropy as the threshold feature of image semantic segmentation and assign weights as deep learning recognition 
(such as entropy weight method), which is widely used in the recognition of objects with obvious particle boundaries, including sand. Tian et al. [23] carried out a triaxial drainage shear test on shell sand from Meizhou Bay, Putian, Fujian Province, and proposed particle size entropy model to measure its crushing rate, and results showed that the grading curve before and after triaxial compression can be characterized by the parameters of particle size entropy model and the particle size entropy parameter of shell sand can better describe its particle crushing behavior. It shows that entropy is a good parameter to describe the system behavior. Zeng et al. [24] introduced the standard basic entropy to characterize the disintegration characteristics of swelling rock. There is a significant negative linear correlation between the standard basic entropy and the disintegration ratio. The standard basic entropy decreases with the increase of the disintegration ratio. It is reasonable to use the standard basic entropy to characterize the particle distribution law, indicating that entropy can be used as a parameter to characterize similar particle distribution law. Loerincz et al. [25] pointed out that the statistical grading entropy of soils $S$ consists of two terms: the base entropy $S 0$ arising from the difference in the width of the statistical cells in the conventional grading curve and the entropy increment $\Delta S$ due to the mixing of the fractions. Laslo [26] pointed out that calculating grain distribution entropy is not "magic" with mathematics: it simply helps put the expected behavior of soils into a different perspective and promotes orientation for classifying soils according to a new parameter related to grain movement. Versaci et al. [27] present a new fuzzy edge detector based on both fuzzy divergence (thought and proved to be a distance) and fuzzy entropy minimization for the thresholding substep in grayscale images. The above works of Lrincz, Laslo, and Versaci illustrate mathematically that the entropy calculation of sand can reflect the distribution law and characteristics of soil particles. However, there is no further correlation analysis with the compactness, and there is no research on the identification of sand core samples after artificial disturbance in the survey site. In order to realize the rapid detection of sand compactness in the field of engineering investigation, it is necessary to take the disturbed sand core sample as the research object. Through the collection of sand core photos, classification, calibration, and segmentation are carried out according to the general image recognition standard, the core image database is established, the calculation model based on texture entropy is established, and the influence law of entropy and compactness is obtained through statistical analysis. There are two kinds of entropy calculation algorithms: one dimension entropy and two-dimensional entropy. This paper focuses on the comparative analysis of the advantages and disadvantages of the two entropy characteristics combined with the experimental results.

Therefore, the author established a database based on the classification and calibration of sand with different compactness collected from the engineering site, analyzed the influence of compactness on it by using image entropy as the main feature, and quantitatively characterized the visual features of the core sample surface with the help of entropy.
Theoretically, it has more advantages than the previous single color or texture feature recognition and has lower computational complexity. It is easy to be applied in engineering practice and provides a new method for rapid identification of core samples in engineering investigation. It is necessary to explore the visual characteristics of sand core samples with different compactness.

\section{Image Features of Sand Core Samples}

\subsection{Generalization of the Image Characteristics of Sand Core} Samples. Relying on the geotechnical engineering investigation projects, statistical analysis of more than 5000 drilling soil layers was made and it was found that the drilling soil samples on-site are generally columnar, as shown in Figure 1 . The stratum structure has a certain degree of primary structure due to the deposition, cycle, and tectonic geological process. Sandy soil belongs to a kind of soil, which is a quartz mineral aggregate, with obvious grain sense, some of which contain a small amount of clay or no cohesion. When the moisture content of soil sample is high, the core sample of loose sand is often not columnar. Some sand cores with fewer clay particles are columnar or massive after drilling, as shown in Figure 2. Sand samples include different sizes, such as gravel, cobble, and coarse sand, maybe in river, lacustrine, and fluvial facies areas, with poor sorting. When there is more cohesive soil, the sand core sample is similar to the hard soil sample, occasionally short column and fragment. In addition, due to the difficulty of sand core drilling, the core drilling process is often accompanied by water injection drilling. The more loose the sand core disturbed by construction is, the more obvious the changes of shape and surface texture will occur. Therefore, when sand is disturbed during coring, there is a close correlation between the grain texture and compactness of the core column surface. The criterion of sand compactness is in accordance with the code for geotechnical engineering investigation. According to the Chinese code for geotechnical engineering investigation (GB 50021-2001), the standard penetration blow number $N_{0} \leq 10$ is loose, $10<N_{0} \leq 15$ is slightly dense, $15<N_{0} \leq 30$ is medium dense, and $N_{0}>30$ is dense.

\subsection{Experimental Design of Image Features of Sand Core Based} on Entropy. The grain texture of sand is essentially a measure of the disorder degree of different size particles. In the information theory, entropy is a measure of the disorder degree of the system. The surface particles of sand can be regarded as a system, the effective information provided by the system data can be measured, and the weight can be determined by entropy. According to iconology theory [2], grayscale uses black tone to represent the object, black is used as the reference color, and black with different saturation is used to display the image. Each gray object has a brightness value from $0 \%$ (white) to $100 \%$ (black), and gray discontinuity appears at the edge of the object. It is reasonable to use entropy to represent texture feature of sand surface.

Therefore, the one-dimensional entropy of image is introduced to represent the information contained in the 


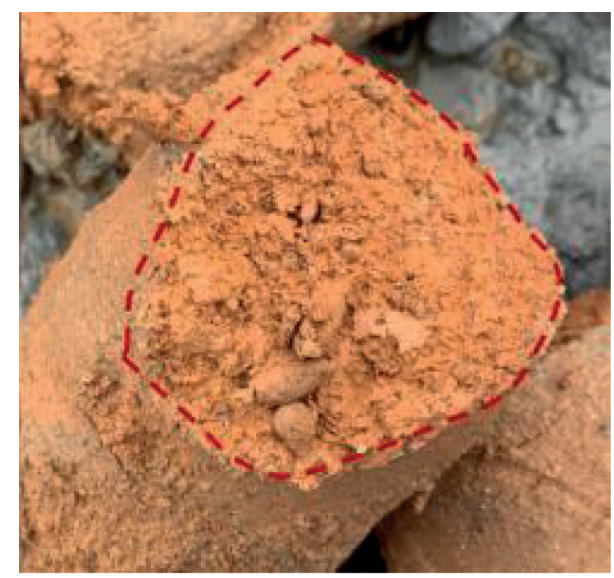

FIGURE 1: Main characteristics of soil core description.

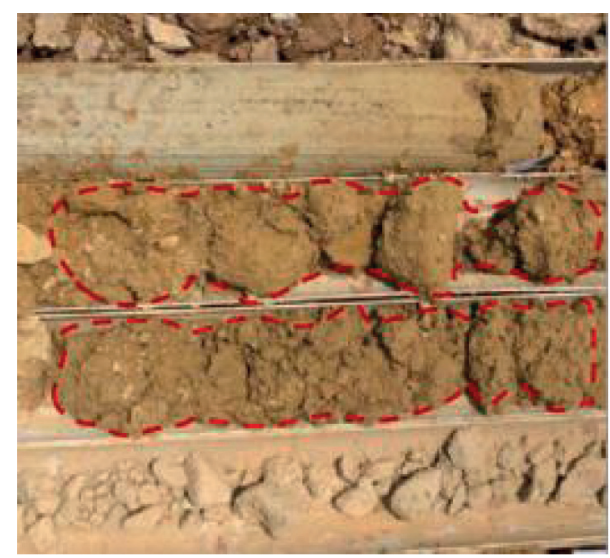

FIGURE 2: Core drilling characteristics of sand sample.

aggregation feature of gray distribution in the image, and the numerical solution of one-dimensional entropy is as shown in equation (1).

$$
H_{1}=-\sum_{i=0}^{255} P_{i} \lg P_{i} .
$$

Based on the one-dimensional entropy, the feature quantity which can reflect the spatial characteristics of gray distribution is introduced to form the two-dimensional entropy of the image. The mean value of neighborhood grayscale of the image is selected as spatial feature quantity of grayscale distribution, and the pixel grayscale of the image forms a feature binary, which is recorded as $(i, j)$, where $i$ represents the grayscale value of the pixel $(0 \leq i \leq 255)$ and $j$ represents the mean value of neighborhood grayscale $(0 \leq j \leq 255)$ :

$$
P_{i j}=\frac{f(i, j)}{N^{2}}
$$

The above formula can reflect the comprehensive characteristics of the gray value of a pixel position and the gray distribution of the surrounding pixels, where $f(i, j)$ is the frequency of the feature binary $(i, j)$ and $N$ is the scale of the image. The formula for calculating the two-dimensional gray entropy of the image is

$$
H_{2}=-\sum_{i} \sum_{j} P_{i, j} \lg P_{i, j}
$$

In order to find out the interaction law between particle entropy and density of sand core samples and fully consider the disturbance effect of engineering drilling construction on sand, we combine with the actual investigation project and collect the sand core samples images obtained from drilling construction. According to the survey report, the loose, slightly dense, and medium dense sand core samples are classified, and 60 groups of different compactness are selected as data analysis samples. The entropy calculation algorithm is compiled by Python to calculate the one-dimensional and two-dimensional gray entropy of the image, respectively, the influence of different compactness on the entropy was discussed, and the technical route is shown in Figure 3.

\section{Engineering Case Analysis}

3.1. Engineering Geological Conditions. The total land area of the project is about $500000 \mathrm{~m}^{2}$. The total construction area is about 1.0183 million square meters. It is planned to build 42 high-rise commercial residential buildings, with 1 2 storey basements. The terrain of the site is relatively flat, and the overall terrain is high in the north and low in the south, belonging to alluvial plain geomorphic unit. The overburden of the site is mainly quaternary artificial fill, alluvial proluvial silting argillaceous soil, silty clay, sand layer, and residual soil layer. It is mainly distributed in the structure of finegrained soil in the upper part and coarse-grained soil in the lower part. In some sections, silty clay and sandy soil are interlaced into layers. The properties of sandy soil are loose, slightly dense, and medium dense, and the uniformity is poor. The drilling layout plan of the project is shown in Figure 4.

\subsection{Surface Entropy Characteristics of Sand Core Samples}

3.2.1. One-Dimensional Entropy Calculation of Sand Core. The one-dimensional entropy and two-dimensional entropy of 60 groups of sand core samples with different properties are calculated, respectively. Firstly, the control variable method is used to calibrate the optical distortion of all core samples. Then, $200 \times 100$ pixels are intercepted in the image. In order to highlight the characteristics of the image more intensively, only the core sample is intercepted when making the sample data set, which ensures that the texture recognition process is not interfered with by the objects around the core sample. The test mainly selects fine sand as the research object and analyzes the loose sand sample, slightly dense sand sample, and medium dense sand sample. The data samples are selected from the sand images of ZK4, ZK11, ZK12, ZK24, ZK38, ZK39, ZK46, ZK54, ZK57, ZK63, ZK66, ZK77, ZK84, and other boreholes with sand layer. The sample data image is shown in Figure 5. 


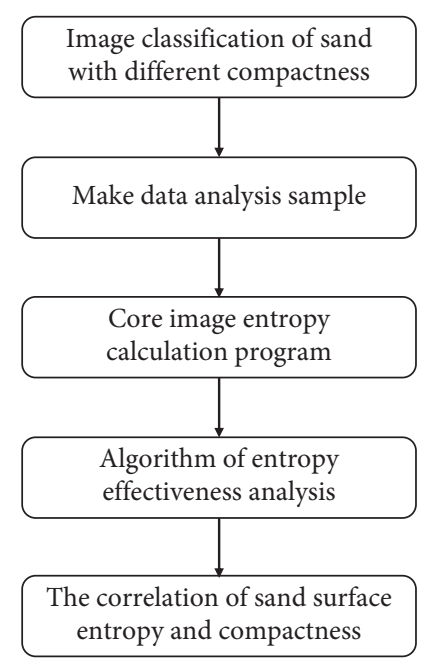

Figure 3: Correlation analysis method.

3.2.2. Calculation of Gray Entropy of Sand Core. Combined with Python language, the entropy calculation algorithm is compiled to calculate the one-dimensional and two-dimensional gray entropy of the above 60 groups of sand core images with different compactness. Taking the number of experimental groups as the independent variable and gray entropy as the dependent variable, the entropy change curves of loose, slightly dense, and medium dense sand are obtained, and the trend lines are obtained by linear regression fitting. The curves are shown in Figure 6 8.

\subsection{Effect of Different Compactness on the Entropy of Sand} Core Samples. Figure 9 is the one-dimensional entropy analysis curve of 20 groups of loose, slightly dense, and medium dense sands in each data sample. From the distribution of trend line, it can be seen that the entropy value of medium dense sands is the largest, while the trend lines of slightly dense and loose sands intersect each other, so it does not well distinguish the two kinds of sand cores. Because the calculation of one-dimensional entropy is mainly based on the aggregation effect of image gray, there is no spatial feature that can reflect the gray change. Some loose sand and slightly dense sand may have large surface space difference, but the surface particles are more uniform, which leads to the difference of entropy value is not obvious.

Figure 10 is a two-dimensional entropy analysis curve based on the feature of rich entropy in gray neighborhood. The two-dimensional entropy analysis of each 20 groups of loose, slightly dense, and medium dense sands is carried out. From the distribution of trend line, it can be seen that the entropy value of medium dense sands is the largest, followed by the entropy value of slightly dense sands and loose sands which is the smallest. In the calculation results, the entropy values of three types of sands have obvious regularity, and the images of loose, slightly dense, and medium dense sands are similar. There is a positive correlation between the twodimensional entropy and density of sand.

Figure 11 shows the distribution of average and median entropy values of sand image. The four curves are as follows: the average value of one-dimensional entropy is No. 1, the average value of two-dimensional entropy is No. 2, the median value of one-dimensional entropy is No. 3, and the median value of two-dimensional entropy is No. 4. The more uniform the grain texture is, the looser the sand is, and the two-dimensional entropy value is smaller. The rougher the grain texture is, the denser the sand is, and the two-dimensional entropy value is greater. The variance of sample data is $0.061,0.302,0.121,0.214,0.034$, and 0.046 , the entropy algorithm is relatively stable.

The entropy of sand with different compactness in the same borehole is analyzed by variance analysis. In loose sand, slightly dense sand, and medium dense sand, four sets of core image entropy of the same borehole are taken for analysis. The maximum and minimum values in the data are removed, and the maximum variance of the two-dimensional entropy of loose sand, slightly dense sand, and medium dense sand images is 0.086, 0.073, and 0.066, respectively; all of them were less than 0.09 , as shown in Figure 12. The average variance of data samples $(0.303,0.214$, and 0.047 ) is different, which shows that the amplification effect of the data variance of two-dimensional entropy is mainly due to the influence of engineering disturbance between different boreholes.

Thus, the influence law of sand core compactness on surface entropy over previous studies is that the entropy of sand image showed that entropy value of medium dense sand is the largest, the latter is slightly dense sand, the loose is the smallest, and the entropy value of loose, slightly dense, and medium dense sand image is positively correlated with the compactness of sand.

In addition, the grayscale of each pixel and the combination of neighboring grayscales have been considered in the calculation process of two-dimensional entropy; the boundary grayscale features of sand image particles are quantified in the calculation. The surface particles of loose, slightly dense, and medium dense sand will form an ordered structure due to their loose to dense structure. The shape structure with gray change boundary increased the roughness of the image, resulting in the increase of entropy. Two- 


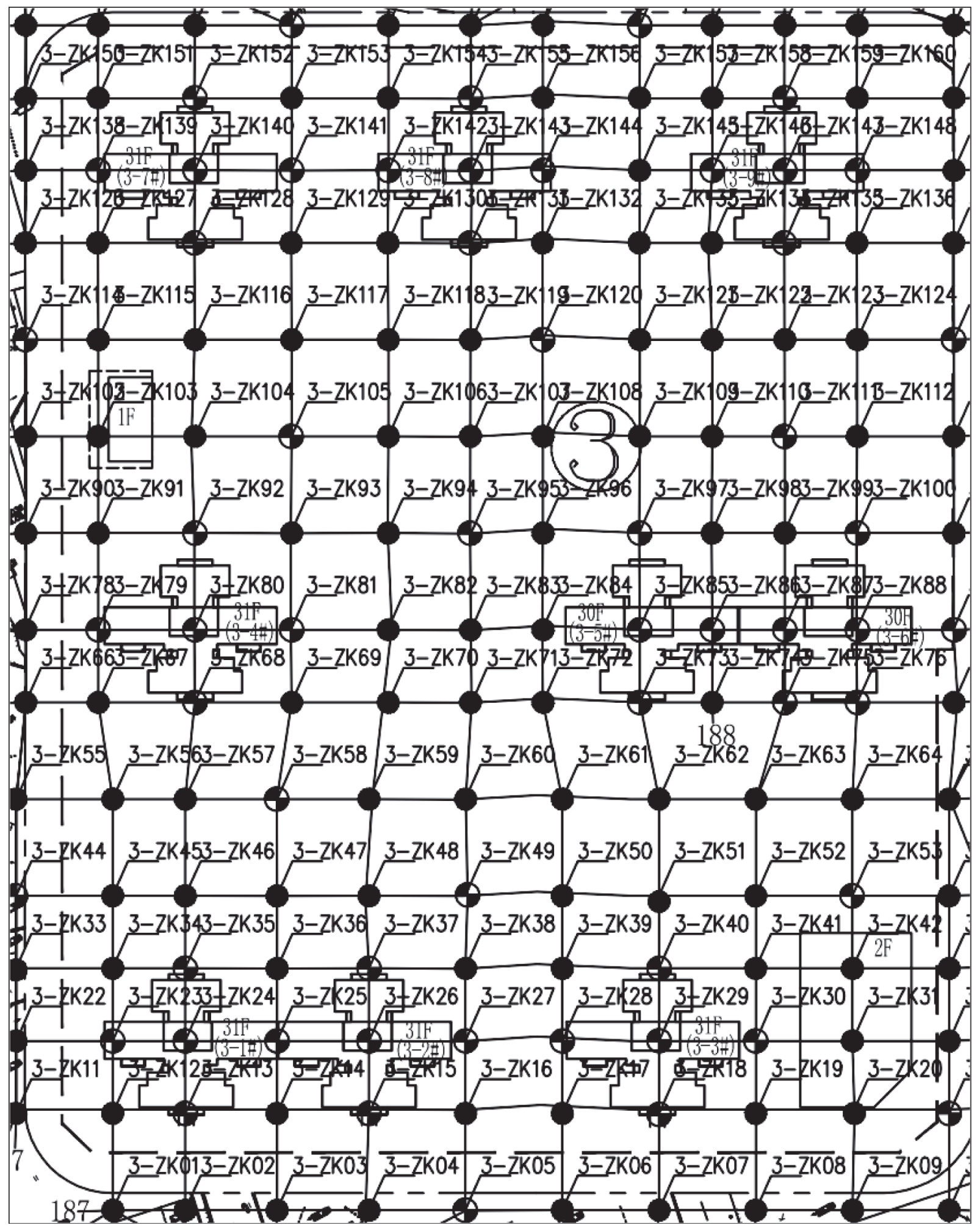

Figure 4: Drilling plan of the project site.

dimensional entropy can more clearly reveal the internal correlation mechanism of the influence of engineering state on the surface structure of sand than one-dimensional entropy and can effectively characterize compactness of sand particles, which has reference value for detection and analysis results of compactness of engineering sand sample. 

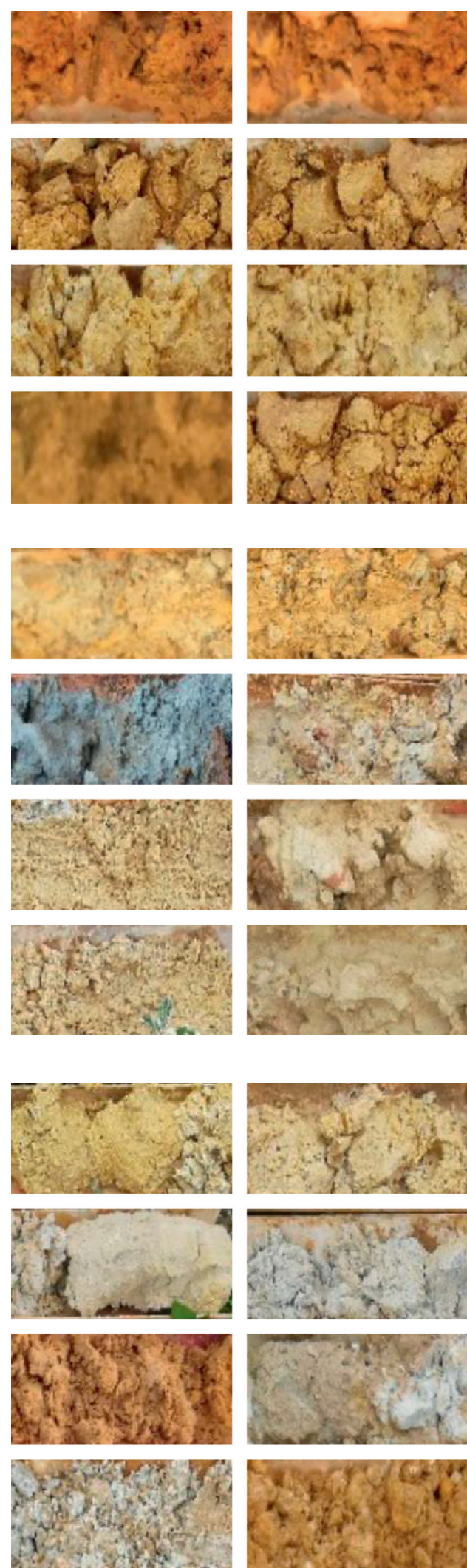
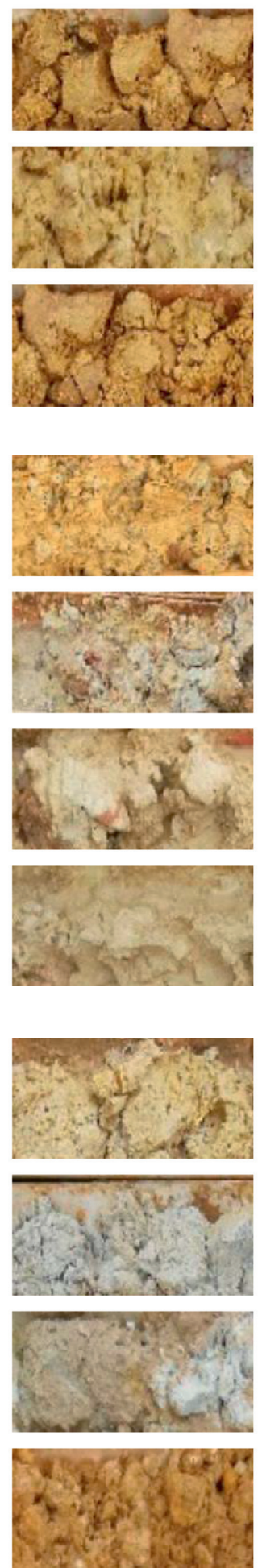
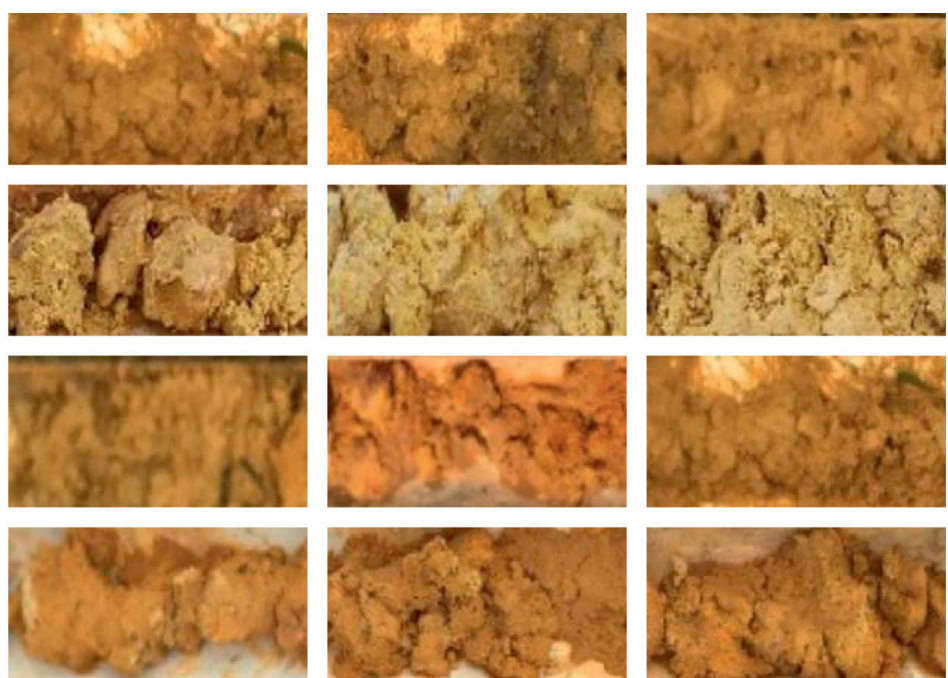

(a)
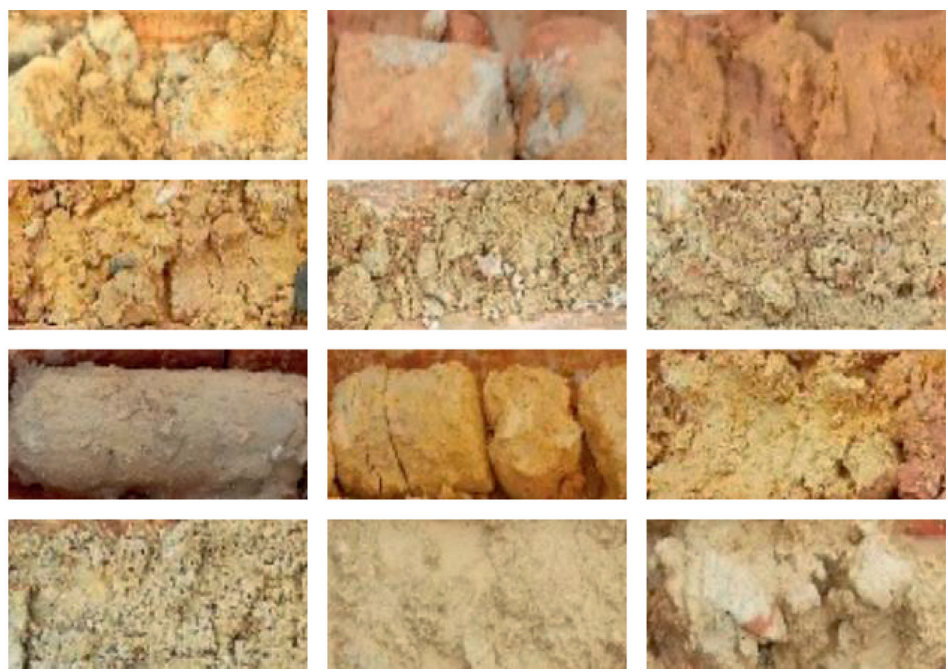

(b)
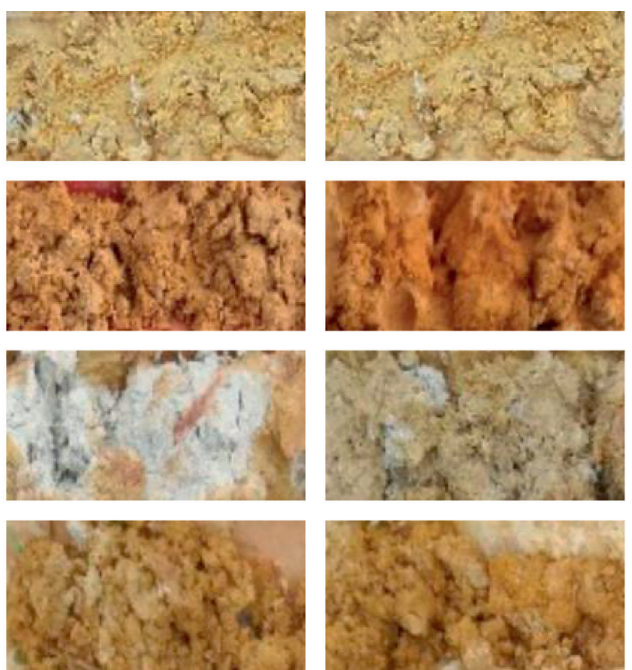

(c)

Figure 5: Sand core sample analysis data sample. (a) Loose sand image. (b) Slightly dense sand image. (c) Medium dense sand image. 


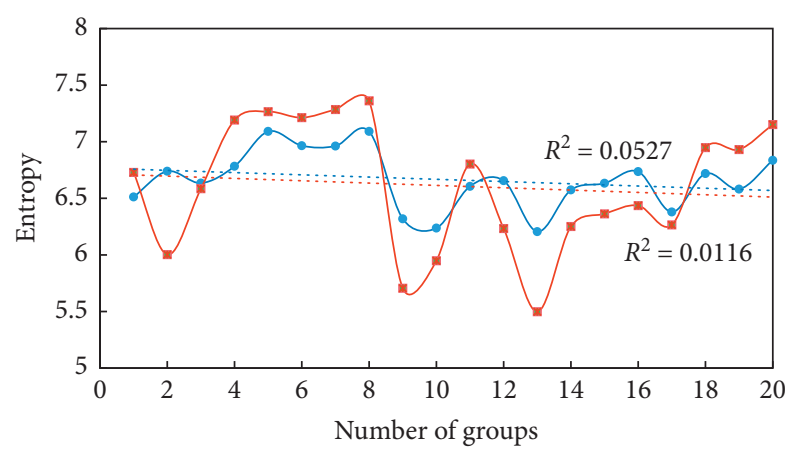

- One-dimensional entropy

_ Two-dimensional entropy

FIgURE 6: Entropy of loose sand.

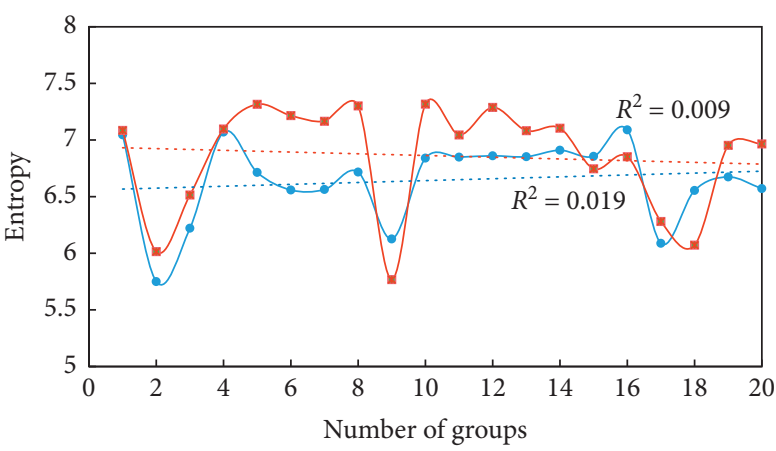

_ One-dimensional entropy

— Two-dimensional entropy

FIGURE 7: Entropy of slightly dense sand.

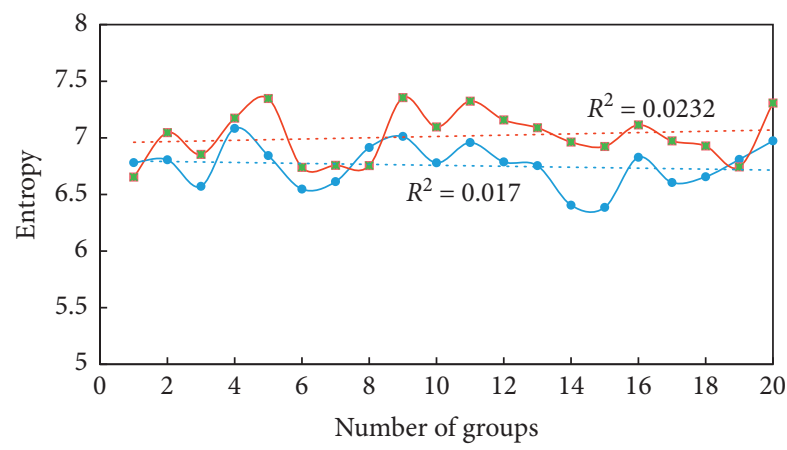

- One-dimensional entropy

_ Two-dimensional entropy

Figure 8: Entropy of middle dense sand.

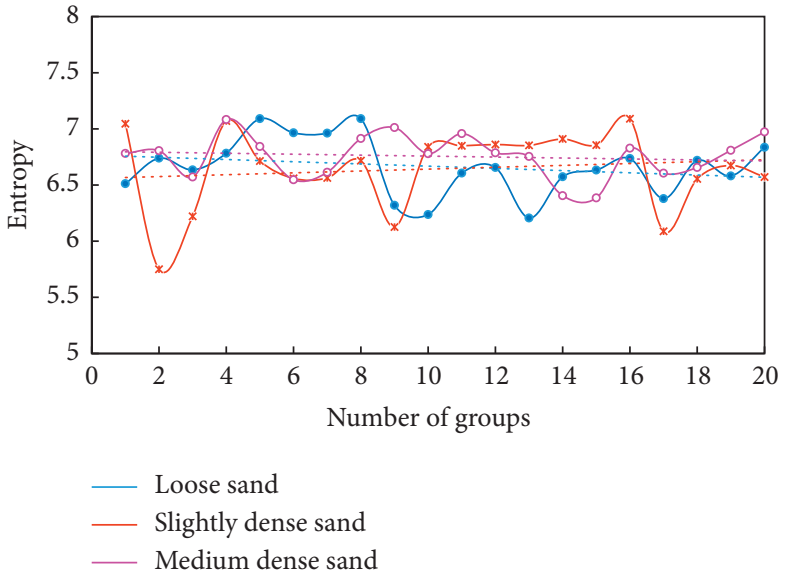

FIGURE 9: Variation of one-dimensional entropy of sand with different compactness.

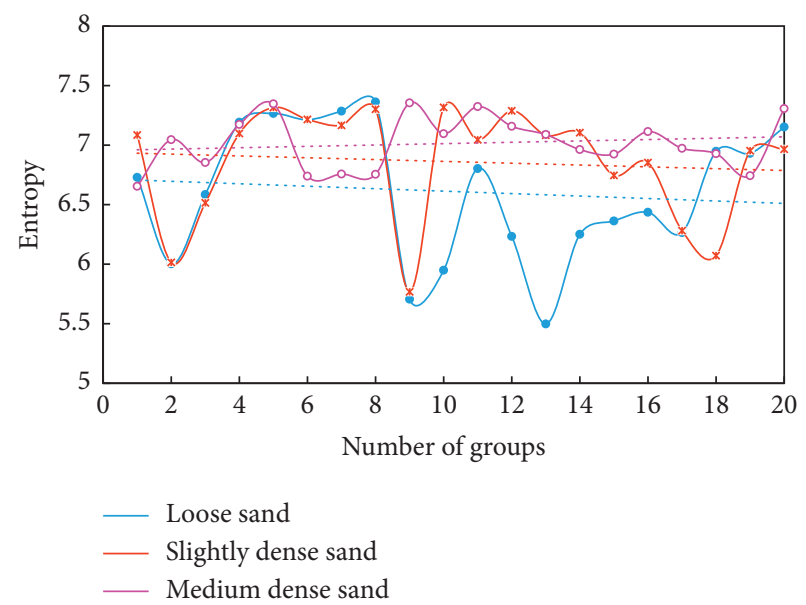

Figure 10: Variation of two-dimensional entropy of sand with different compactness.

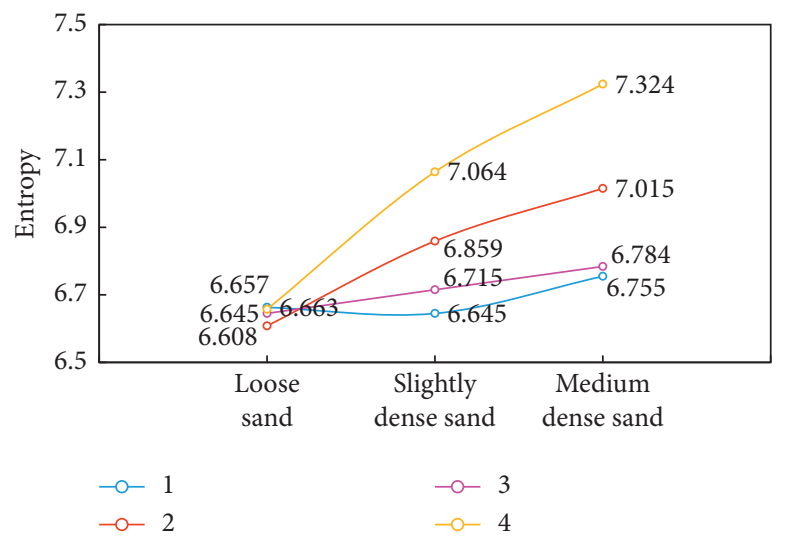

Figure 11: Average and median entropy of sand image with different compactness. 


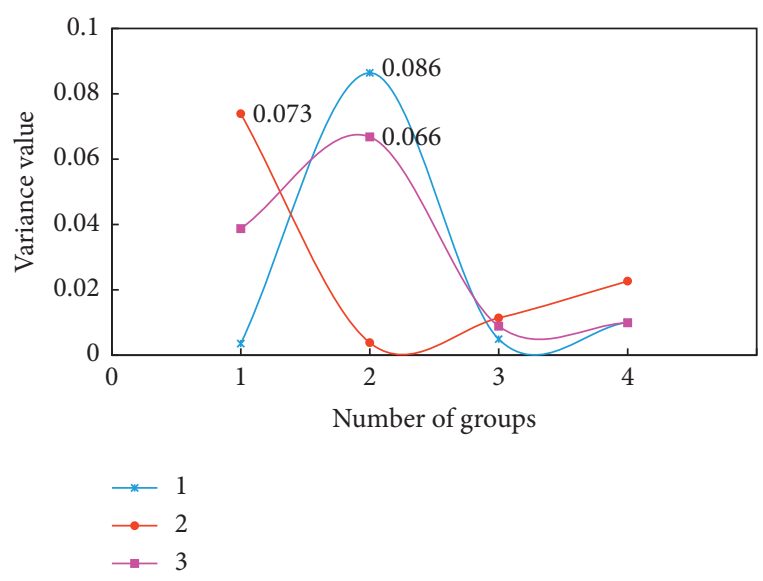

FIGURE 12: Two-dimensional entropy maximum variance analysis of samples from the same borehole.

\section{Conclusion}

The geotechnical investigation environment is regional, and the core sample is easily disturbed by artificial factors. The surface characteristics of sand core samples were generalized in actual investigation projects. Sand images with different compactness were selected. The entropy calculation algorithm was compiled. The one-dimensional and two-dimensional entropy of sample images were calculated, respectively. The influence of density states on entropy was discussed.

(1) Affected by drilling construction and disturbance, the looser the sand core surface particles are, the worse the sorting is and the more irregular the shape characteristics are. After the sand is disturbed during drilling, the grain texture and density of core column surface are related closely.

(2) The entropy of sand image showed that entropy value of medium dense sand is the largest, the latter is slightly dense sand, the loose is the smallest, and the entropy value of loose, slightly dense, and medium dense sand image is positively correlated with the compactness of sand.

(3) The maximum variance of two-dimensional entropy in the same borehole loose sand, slightly dense sand, and medium dense sand images is less than 0.09, showing that the amplification effect of entropy was mainly due to engineering disturbance between the different boreholes.

(4) The data generated by the two-dimensional entropy is small fluctuation and the algorithm is stable and reliable. It is of reference value to the test and analysis results of sand sample density.

(5) The grayscale of pixels and the combination of neighborhood gray have been considered in the calculation of two-dimensional entropy. The boundary gray characteristics of sand image particles are quantified in the calculation. The surface particles of loose, slightly dense, and medium dense sand will form an ordered structure due to their loose to dense structure. The shape structure with gray change boundary increases the roughness of the image, resulting in the increase of entropy. The two-dimensional entropy is clearer than one-dimensional entropy, which reveals the internal correlation mechanism of the influence of engineering state on the surface structure of sand, and more effectively characterizes the density of sand particles.

\section{Data Availability}

The raw/processed data required to reproduce these findings cannot be shared at this time as the data also form part of an ongoing study.

\section{Conflicts of Interest}

The authors declare that they have no conflicts of interest.

\section{Acknowledgments}

This word was supported by the China Postdoctoral Science Foundation Funded Project (2020M682671), Science and Technology Planning Project of Guangzhou (no. 202102020684), Project Funding of Innovation and Entrepreneurship Leading Team in Baiyun District, Guangzhou (no. (2021)-0305), and Science and Technology Progress Fund Project of Guangzhou Institute of Building Science Co. Ltd. (no. 2019Y-KJ02).

\section{References}

[1] Y. Wu, N. Li, X. Wang et al., "Experimental investigation on mechanical behavior and particle crushing of calcareous sand retrieved from South China Sea," Engineering Geology, vol. 280, Article ID 105932, 2021.

[2] Y. Wu, J. Cui, J. Huang, and W. Zhang, "Correlation of critical state strength properties with particle shape and surface fractal dimension of clinker ash," International Journal of Geomechanics, vol. 21, no. 6, Article ID 4021071, 2021.

[3] M. M. He, Z. Q. Zhang, and N. Li, "Deep convolutional neural network-based method for strength parameter prediction of jointed rock mass using drilling logging data," International Journal of Geomechanics, vol. 21, no. 7, 2021.

[4] D. L. Su, H. S. Hu, and X. R. Xie, "Research status of intelligent information technology in geotechnical engineering exploration," Guangzhou Architecture, vol. 47, no. 6, pp. 10-18, 2019.

[5] Y. M. Yang, N. Liu, G. J. Cheng, and X. Qiang, "Clustering analysis of rock images based on spark platform," Journal of Xi'an Shiyou University(Natural Science), vol. 31, no. 6, pp. 114-118, 2016.

[6] C. F. Zhang, L. N. Hao, Y. J. Wang, and Z. Zhang, "An image enhancement and lithology identification method based on landsat8 OLI data," Geology and Exploration, vol. 53, no. 2, pp. 325-333, 2017.

[7] M. M. He, Z. Q. Zhang, J. W. Zhu, N. Li, G. Li, and Y. Chen, "Correlation between the rockburst proneness and friction characteristics of rock materials and a new method for rockburst proneness prediction: field demonstration," Journal 
of Petroleum Science and Engineering, vol. 205, Article ID 108997, 2021.

[8] C. L. Liu, M. X. Tang, H. S. Hu et al., "Model reconstruction and FEM analysis of grouted pile-soil interfaces based on 3D laser scanning technology," Guangzhou Architecture, vol. 47, no. 6, pp. 19-24, 2019.

[9] Q. Rao, H. Wen, W. Yu et al., "Artificial neural network image classification based on super-pixel," Computer and Modernization, vol. 12, pp. 98-101, 2013.

[10] H. Yahong, W. Fei, Z. Yueting, and H. Xiaofei, "Multi-label transfer learning with sparse representation," IEEE Transactions on Circuits and Systems for Video Technology, vol. 20, no. 8 , pp. 1110-1121, 2010

[11] S. Niekum, "Reliable rock detection and classification for autonomous science," CMU thesis, Carnegie Mellon University, Pittsburgh, PA, USA, 2005.

[12] P. Y. Wang and S. H. Wang, "Method for identifying four common rock types of slopes and determining boundary range," Chinese Journal of Geotechnical Engineering, vol. 41, pp. 1-8, 2019.

[13] K. Hong, E. Han, and K. Kang, "Determination of geological strength index of jointed rock mass based on image processing," Journal of Rock Mechanics and Geotechnical Engineering, vol. 9, no. 4, pp. 702-708, 2017.

[14] N. Singh, T. N. Singh, A. Tiwary, and K. M. Sarkar, "Textural identification of basaltic rock mass using image processing and neural network," Computational Geosciences, vol. 14, no. 2, pp. 301-310, 2010.

[15] L. Shu, K. Mcisaac, G. R. Osinski, and R. Francis, "Unsupervised feature learning for autonomous rock image classification," Computers \& Geosciences, vol. 106, pp. 10-17, 2017.

[16] J. F. Zhang, X. J. Zhang, G. S. Yang et al., "A method of rock CT image segmentation and quantification based on clustering algorithm," Journal of Xi'an University of Science and Technology, vol. 36, no. 2, pp. 171-175, 2016.

[17] Y. C. Ke, Z. K. Shi, P. J. Li, and X. Zhang, "Lithological classification and analysis using hyperion hyperspectral data and random forest method," Acta Petrologica Sinica, vol. 34, no. 7, pp. 2181-2188, 2018.

[18] F. C. Meng, Y. H. Zhao, and Z. H. Zheng, "Experimental study of the effect of relative compactness on dynamic shear modulus and damping ratio of clayey sand," China Earthquake Engineering Journal, vol. 43, no. 2, pp. 396-403, 2021.

[19] C. Misko and I. Kenji, "Empirical correlation between SPT Nvalue and relative density for sandy soils," Soils and Foundations, vol. 39, no. 5, pp. 61-71, 1999.

[20] C. Wightman, F. J. Muzzio, and J. Wilder, "A quantitative image analysis method for characterizing mixtures of granular materials," Powder Technology, vol. 89, no. 2, pp. 165-176, 1996.

[21] F. Zou, B. H. Wang, and P. M. Jiang, "Statistics of sand particles based on the digital image technology," Journal of Jiangsu University of Science and Technology (Natural Science Edition), vol. 29, no. 2, pp. 180-185, 2015.

[22] B. Zhou, Statistical Method of Sand Particles Based on Binary Image Processing Technology, Shenzhen University, Shenzhen, China, 2017.

[23] H. Tian, L. W. Kong, and D. Zhao, "Run-of-bank sand," Dictionary Geotechnical Engineering/Wörterbuch GeoTechnik, vol. 36, no. 6, Article ID 1152, 2014.

[24] Z. X. Zeng, L. W. Kong, H. Tian, and J. Li, "Effect of drying and wetting cycles on disintegration behavior of swelling mudstone and its grading entropy characterization," Rock and Soil Mechanics, vol. 38, no. 7, pp. 1983-1989, 2017.
[25] J. Loerincz, E. Imre, M. Galos, and Q. Trang, "Grading entropy variation due to soil crushing," International Journal of Geomechanics, vol. 5, no. 4, pp. 311-319, 2005.

[26] N. Laslo, "Characterization of sand boils with grading entropy," Zbornik Matice srpske za prirodne nauke, vol. 2012, no. 122, pp. 73-88, 2012.

[27] M. Versaci and F. C. Morabito, "Image edge detection: a new approach based on fuzzy entropy and fuzzy divergence," International Journal of Fuzzy Systems, vol. 23, pp. 1-19, 2021. 